\title{
Correlation between 2MASS and DENIS data
}

\author{
A. Cabrera-Lavers ${ }^{1}$ and F. Garzón ${ }^{1,2}$ \\ 1 Instituto de Astrofísica de Canarias, 38200 La Laguna, Tenerife, Spain \\ 2 Departamento de Astrofísica, Universidad de La Laguna, Tenerife, Spain \\ Received 7 January 2003 / Accepted 25 February 2003

\begin{abstract}
We have cross-correlated 2MASS and DENIS point sources in 17 galactic fields in order to find systematic astrometric or photometric shifts between the surveys. No significant differences have been found between the data of the two filters in common $\left(J\right.$ and $\left.K_{\mathrm{s}}\right)$. This allows to combine data from the two surveys for a further analysis of galactic structure via star counts.
\end{abstract}

Key words. surveys - infrared: stars - stars: statistics

\section{Introduction}

IR Deep Galactic surveys have become in the last decade a powerful tool to investigate Galactic structure and interstellar extinction via star counts (Garzón et al. 1993; Hammersley et al. 1994; López-Corredoira et al. 2000, 2002). The Two Micron Galactic All Sky Survey (2MASS) (Struskie et al. 1997) and the Deep Near Infrared Survey of the Southern Sky (DENIS) (Epchtein et al. 1997; Fouqué et al. 2000) have been scanning the sky for the last 5 years, acquiring a large amount of data that are starting to be accessible to the scientific community. At present, 2MASS and DENIS data are not totally available. The 2MASS project provides public access for $47 \%$ of the Sky with its Second Incremental Release. On the other hand, $80 \%$ of DENIS observations have been reduced at the Paris Data Analysis Centre (PDAC) and 60\% of data are accessible to date but they only refer to the Southern Sky. A global study of galactic structure via star counts will not be possible until the All Sky Release of 2MASS which will provide $J, H$, and $K_{\mathrm{s}}$ band photometry for millions of galaxies and nearly a half-billion stars (this release is expected to be finished in the first half of 2003). However, combining data from 2MASS and DENIS will provide a full and partially redundant coverage of the Galaxy, permitting a better analysis of Galactic structure. Our purpose in this paper is to compare 2MASS and DENIS star counts to allow more or less automatic blending of the two data sets, supporting future work in galactic structure via star counts. We have found in the literature some similar analyses with data from 2MASS and DENIS, however they are restricted to very specific areas of the sky (Delmotte et al. 2001; Carpenter 2001; Schultheis \& Glass 2001). On the contrary, our analysis includes a large number of galactic fields, covering as much longitude range as possible with the available data of the surveys.

Send offprint requests to: A. Cabrera-Lavers, e-mail: acabrera@ll.iac.es

\section{Data}

The 2MASS data set that we have used here is the second incremental data release (2IDR) of the 2MASS project (http: //www . ipac. caltech. edu/2mass) that covers 47 per cent of the sky. Selected DENIS data are available through the web server at the Centre de Données Astronomiques de Strasbourg (http://cdsweb.u-strasbg.fr/denis.html), except some fields: $l=230^{\circ} b=3^{\circ}, l=235^{\circ} b=-6^{\circ}$, $l=240^{\circ} b=0^{\circ}$ and $l=245^{\circ} b=9^{\circ}$, whose data were extracted directly from the private section of the Paris Data Analysis Center (PDAC), which soon will become available to the public. For the selected fields, queries were made around a given position (center and radius, where the center was specified in $\mathrm{J} 2000$ coordinates). We chose a $0.5^{\circ}$ radius for the source extraction, with the central positions selected in 1 degree steps. In this way, part of the selected field is not covered but it does not affect the study as we obtained enough common sources in each field. Central coordinates were translated into Galactic coordinates for consistency with the organization of the 2MASS data set.

\section{Matching 2MASS and DENIS sources}

We selected 17 regions where both 2MASS and DENIS data are available. Most fields are located near the Galactic plane along the outer Galactic disc $\left(220^{\circ}<l<275^{\circ},|b|<12^{\circ}\right)$. There are also two inner galactic fields, in order to include high extinction regions in the comparison. In each field, we consider only sources detected in all the filters $\left(I J K_{\mathrm{s}}\right.$ in DENIS, and $J H K_{\mathrm{s}}$ in $2 \mathrm{MASS}$ ). After rejecting those sources not detected in all the filters, we cross-correlated the two catalogues, using only astrometric criteria, and obtained mean astrometric and photometric differences between the common sources. Table 1 summarizes the fields selected for comparison between 2MASS and DENIS data. Central coordinates (in the galactic 
Table 1. Selected regions where data from 2MASS and DENIS were cross-correlated.

\begin{tabular}{ccccccccccc}
\hline \hline$l(\mathrm{deg})$ & $b(\mathrm{deg})$ & RA $(\mathrm{J} 2000)$ & Dec $(\mathrm{J} 2000)$ & $N$ & area & $r$ & $<\Delta J>$ & $\sigma_{J}$ & $<\Delta K>$ & $\sigma_{K}$ \\
\hline 230 & -3 & $+07: 12: 17.04$ & $-16: 32: 32.64$ & 2518 & 2.85 & $2^{\prime \prime}$ & $0.016 \pm 0.003$ & 0.13 & $-0.022 \pm 0.003$ & 0.15 \\
230 & 3 & $+07: 34: 12.15$ & $-13: 42: 33.54$ & 2069 & 2.39 & $1^{\prime \prime}$ & $-0.035 \pm 0.002$ & 0.09 & $0.027 \pm 0.004$ & 0.17 \\
230 & 6 & $+07: 44: 57.31$ & $-12: 14: 48.71$ & 259 & 0.55 & $1^{\prime \prime}$ & $0.058 \pm 0.005$ & 0.07 & $0.043 \pm 0.005$ & 0.08 \\
230 & 12 & $+08: 06: 06.97$ & $-09: 14: 52.33$ & 827 & 3.01 & $1^{\prime \prime}$ & $-0.027 \pm 0.004$ & 0.10 & $0.027 \pm 0.007$ & 0.19 \\
235 & -6 & $+07: 10: 37.61$ & $-22: 20: 47.47$ & 1352 & 0.52 & $1^{\prime \prime}$ & $0.017 \pm 0.006$ & 0.07 & $-0.009 \pm 0.005$ & 0.14 \\
235 & 0 & $+07: 33: 19.91$ & $-19: 32: 04.48$ & 2035 & 2.26 & $1^{\prime \prime}$ & $-0.027 \pm 0.004$ & 0.10 & $-0.007 \pm 0.004$ & 0.16 \\
240 & 0 & $+07: 43: 54.33$ & $-23: 53: 25.38$ & 2118 & 0.62 & $1^{\prime \prime}$ & $-0.006 \pm 0.002$ & 0.08 & $0.018 \pm 0.002$ & 0.09 \\
245 & -9 & $+07: 18: 39.64$ & $-32: 32: 59.27$ & 896 & 2.50 & $1^{\prime \prime}$ & $-0.052 \pm 0.004$ & 0.12 & $-0.031 \pm 0.006$ & 0.17 \\
245 & 9 & $+08: 28: 44.07$ & $-23: 17: 20.42$ & 632 & 0.30 & $1^{\prime \prime}$ & $0.022 \pm 0.003$ & 0.08 & $0.020 \pm 0.005$ & 0.11 \\
250 & 0 & $+08: 07: 27.88$ & $-32: 26: 32.87$ & 1171 & 2.12 & $1^{\prime \prime}$ & $0.021 \pm 0.002$ & 0.08 & $-0.024 \pm 0.006$ & 0.20 \\
250 & 3 & $+08: 19: 13.11$ & $-30: 47: 14.38$ & 537 & 0.51 & $1^{\prime \prime}$ & $-0.064 \pm 0.005$ & 0.11 & $-0.021 \pm 0.006$ & 0.13 \\
255 & 0 & $+08: 20: 55.00$ & $-36: 36: 20.26$ & 333 & 0.89 & $1^{\prime \prime}$ & $-0.044 \pm 0.006$ & 0.11 & $-0.014 \pm 0.008$ & 0.14 \\
275 & -9 & $+08: 48: 16.77$ & $-57: 59: 25.55$ & 465 & 2.16 & $1^{\prime \prime}$ & $-0.003 \pm 0.004$ & 0.09 & $0.004 \pm 0.008$ & 0.17 \\
290 & -6 & $+10: 41: 19.04$ & $-65: 33: 01.85$ & 3011 & 2.71 & $4^{\prime \prime}$ & $0.002 \pm 0.004$ & 0.20 & $0.002 \pm 0.004$ & 0.21 \\
290 & -3 & $+10: 53: 59.26$ & $-62: 53: 04.68$ & 5319 & 2.71 & $4^{\prime \prime}$ & $0.017 \pm 0.004$ & 0.25 & $-0.001 \pm 0.003$ & 0.24 \\
354 & 0 & $+17: 30: 33.14$ & $-34: 00: 18.04$ & 1555 & 0.94 & $2^{\prime \prime}$ & $0.107 \pm 0.008$ & 0.29 & $0.096 \pm 0.008$ & 0.33 \\
359 & 0 & $+17: 43: 13.12$ & $-29: 47: 18.16$ & 1106 & 0.72 & $1^{\prime \prime}$ & $0.103 \pm 0.007$ & 0.24 & $0.047 \pm 0.007$ & 0.24 \\
\hline
\end{tabular}

and equatorial system) are shown in Cols. 1-4. Column 5 gives the number of matched sources. The total area in $\mathrm{deg}^{2}$ (defined by the DENIS data because its smaller coverage) and the search radius used in the cross-correlation are also given in Cols. 6 and 7. Mean photometric differences (2MASS-DENIS) and the standard deviation of the resulting distribution are shown in Cols. 8-11.

As the 2MASS survey reaches almost one magnitude fainter than DENIS, we computed astrometric and photometric differences for sources brighter than 14 in $J$ and 13 in $K_{\mathrm{s}}$ well above the limiting magnitudes of both surveys. In the inner galactic fields, those limiting magnitudes are brighter (13 in $J$ and 11 in $K_{\mathrm{s}}$ ) as DENIS is complete to about 13.5 in $J$ and 12 in $K_{\mathrm{s}}$ (Schultheis \& Glass 2001). While 2MASS default magnitudes are specified for each source (see 2MASS project web page for details in the Data Processing, http: //www . ipac . caltech.edu/2mass), the DENIS catalogue provides different magnitudes for the same source in each band of the survey. In this case, we consider the MAG_AUTO magnitude, an automatic (Kron type) scaling elliptical aperture magnitude, for public data coming from CDS while we used the PSF magnitudes in those fields where data were taken from PDAC.

We cross-correlated data from the DENIS survey with the 2MASS data by using an initial searching box of 1". Stars with absolute astrometric differences less than $1^{\prime \prime}$ both in right ascension and declination were extracted from both catalogues and assumed to be the same star. With those identifications we obtained the photometric differences in $J$ and $K_{\mathrm{s}}$ bands. Both catalogues were calibrated on the USNO-A2.0 catalogue with an astrometric accuracy of $0.5^{\prime \prime}$, so we consider a searching box of $1^{\prime \prime}$ to match common stars in both data sets.

However, there were some fields where it was necessary to increase the search radius to find a reasonable number of identifications: in two cases up to $2^{\prime \prime}$, but there are also two fields with differences up to $4^{\prime \prime}$ (see Table 1).

\subsection{Inner galaxy}

There are two fields located in the inner Galaxy. In those fields, we are limited by source confusion and the magnitudes up to which comparisons between the two surveys are brighter than in the rest of the fields. As explained before, we chose limiting magnitudes of 13 in $J$ and 11 in $K_{\mathrm{s}}$, but we also have to decide if the source confusion is strong enough to need a shorter search radius in the cross-correlation. We tried with different search radii in order to identify the one that provides a better crosscorrelation without any redundant source detection (stars with more than one possible counterpart in 2MASS data). We obtain cross-correlations with search radii in the range $\left[0.5^{\prime \prime}-30^{\prime \prime}\right]$, obtaining an optimal detection for a value no larger than $2 "$. For 3 " there are $1 \%$ of sources with more than one possible counterpart, but 2 " provides only one cross-identification in 2MASS data for each DENIS source (see Fig. 1).

\section{Astrometric and photometric differences}

The global astrometric differences are shown in Fig. 2. Each point represents one association between 2MASS and DENIS sources. As was expected, the points are centred around $(0,0)$, with mean astrometric differences of $\triangle \mathrm{RA}=00^{\prime \prime} 10 \pm 0 .{ }^{\prime} 01$ $(\sigma=0.42)$ and $\Delta \mathrm{Dec}=00^{\prime \prime} 20 \pm 0.0^{\prime} 01(\sigma=0.31)$. Once source identification is done, we compared the $J$ and $K_{\mathrm{s}}$ magnitudes for each star. As it is shown in Figs. 3, 4, there is no significant shift in the magnitudes, with a distribution of differences with $\langle\Delta J>=0.003 \pm 0.001$ (with $\sigma=0.10$ ) and $<\Delta K_{\mathrm{s}}>=-0.004 \pm 0.002$ (with $\sigma=0.14$ ). Figures 3-5 are obtained combining stars from the whole data set. The mean 


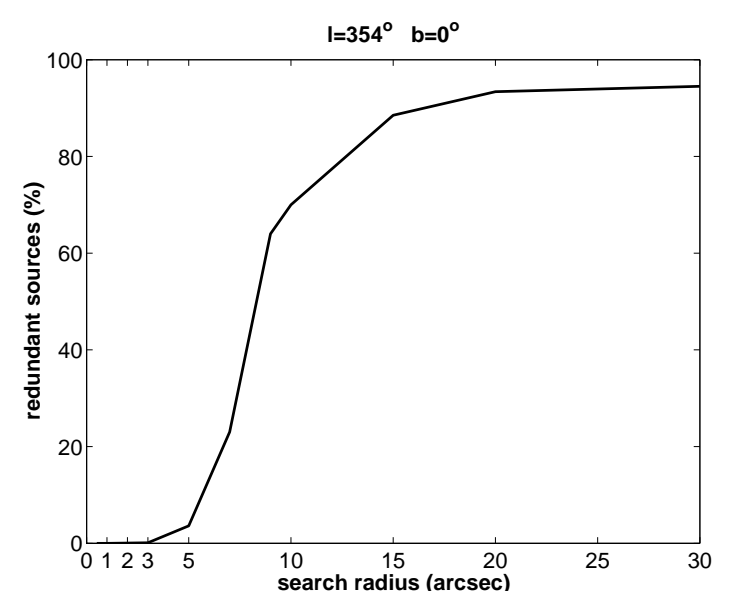

Fig. 1. Percentage of DENIS sources with more than one possible 2MASS counterpart as function of the search radius used, for the field $l=354^{\circ} b=0^{\circ}$. For a search radius shorter than $2^{\prime \prime}$ there are no double identifications while for a search radius larger than $10^{\prime \prime}$ most of the sources present more than one counterpart.

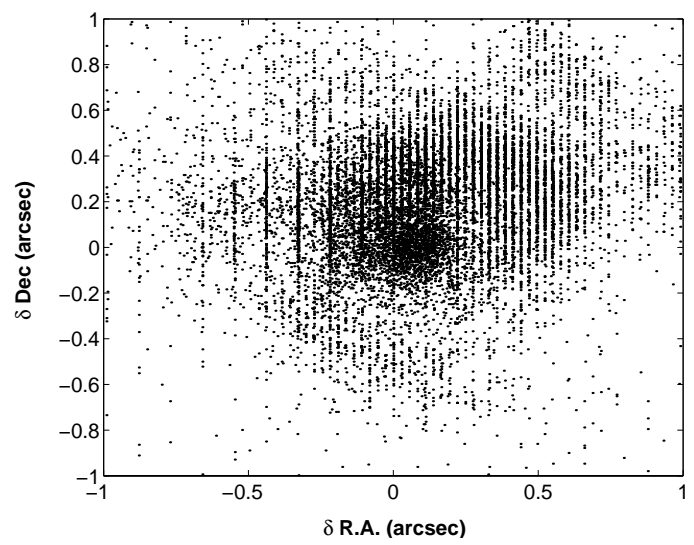

Fig. 2. Astrometric differences between DENIS and 2MASS matched sources, combining all those fields where a searching box of $1^{\prime \prime}$ was used.

differences for each field in particular are shown in Table 1. The results are very similar to those of Delmotte et al. (2001) for the Magellanic Clouds and Schultheis \& Glass (2001) for Baade's Windows, who obtained photometric differences between the surveys of less than 0.10 mag in both filters.

\subsection{Colour distribution}

We also fitted $(J-K)$ colours between the two data sets (see Fig. 5). We previously omitted from the fit those stars with photometric differences greater than $0.5 \mathrm{mag}$, that we consider too high to represent the same star; these probably appear as a result of a wrong cross-correlation between the data sets, or as an effect of variability of some of those stars. In any case, it is not a critical issue in the linear fit as we reject less than $3 \%$ of the stars (from a total number of 26253 stars). The linear fit obtained is:

$$
\begin{aligned}
(J-K)_{2 \mathrm{MASS}}= & (1.020 \pm 0.025)(J-K)_{\text {DENIS }} \\
& +(-0.009 \pm 0.001) .
\end{aligned}
$$
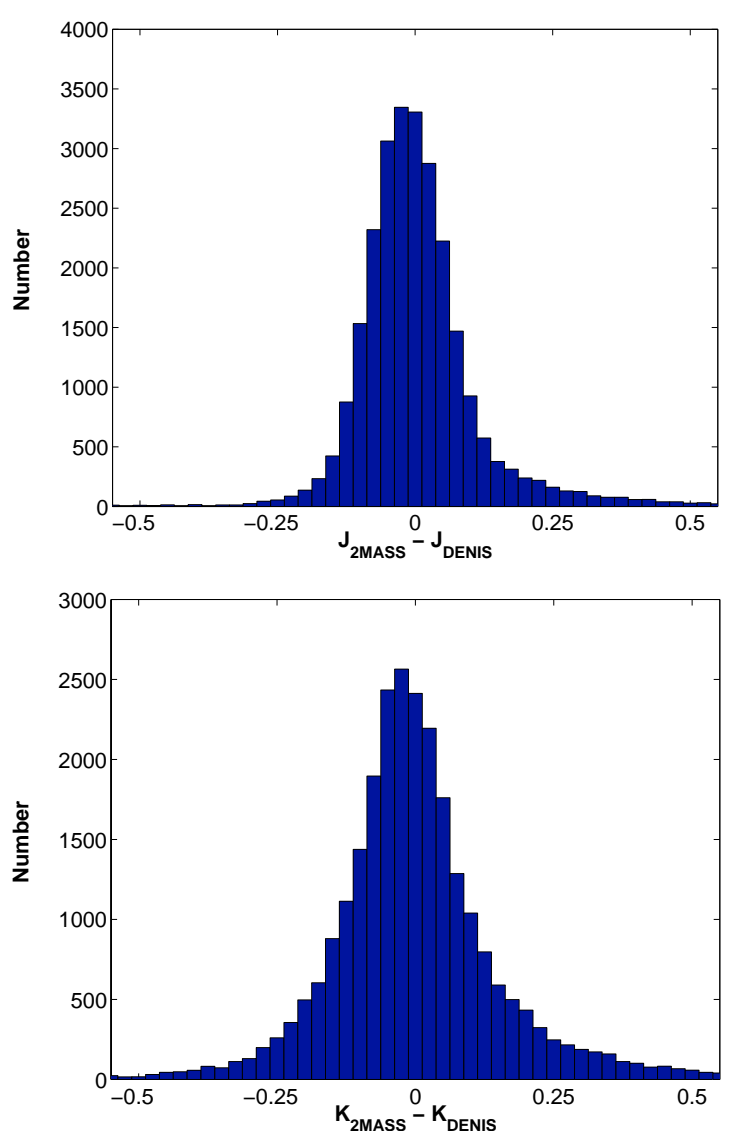

Fig. 3. Histograms of differences between DENIS and 2MASS magnitudes.

This result is consistent, within the error bars, with the previous result of Carpenter (2001), who obtained a slope for the linear fit of 0.981 with 190 stars.

\section{Differences in DENIS multistrip fields}

The data in the different DENIS fields used in this paper come from several individual adjacent strips, observed on different nights, not necessarily consecutive, and some of them were reobserved after the data quality assessment showed unacceptable results. In these latter cases, we have always used the latest strip number incorporated to the data base. All these combined effect may induced systematic effects in the fields which we will try to address in this section.

To this end, we have analyzed two fields in more detail by separating the stars coming from different strips. The results are shown in Fig. 6 in the form of histograms of photometric differences (2MASS - DENIS) for the combined field and for the individual strips. There are no remarkable differences in the shape or in the location of individual histograms with respect to the global ones, which may indicate systematic effects. The numerical results of the individual correlations, strip by strip, between 2MASS and DENIS can be found in Table 2, which again shows no noticeable effects. 
Table 2. Mean photometric differences for the isolated strips.

\begin{tabular}{cccccccc}
\hline \hline$l(\mathrm{deg})$ & $b(\mathrm{deg})$ & DENIS strips & $N$ & $\langle\Delta J\rangle$ & $\sigma_{J}$ & $\langle\Delta K\rangle$ & $\sigma_{K}$ \\
\hline 235 & -6 & 3479 & 419 & $0.054 \pm 0.003$ & 0.06 & $0.034 \pm 0.005$ & 0.11 \\
235 & -6 & 8918 & 405 & $0.007 \pm 0.003$ & 0.07 & $0.017 \pm 0.005$ & 0.10 \\
235 & -6 & 8924 & 430 & $0.015 \pm 0.004$ & 0.07 & $0.039 \pm 0.004$ & 0.09 \\
240 & 0 & 3533 & 390 & $0.014 \pm 0.003$ & 0.06 & $0.031 \pm 0.006$ & 0.11 \\
240 & 0 & 3705 & 299 & $-0.025 \pm 0.005$ & 0.07 & $0.011 \pm 0.007$ & 0.10 \\
240 & 0 & 8963 & 462 & $0.014 \pm 0.004$ & 0.09 & $0.025 \pm 0.004$ & 0.08 \\
\hline
\end{tabular}
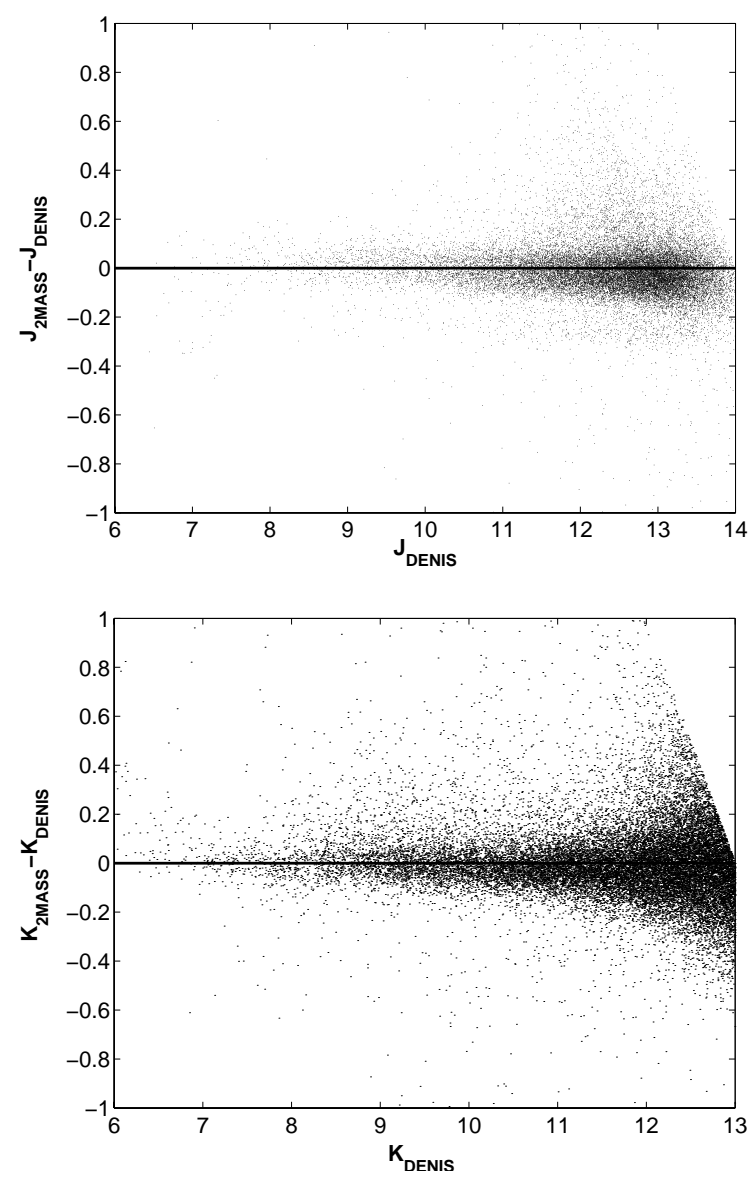

Fig. 4. (Above) $J_{2 \mathrm{MASS}}-J_{\text {DENIS }}$ as a function of $J_{\text {DENIS }}$. (Below) $K_{2 \mathrm{MASS}}-K_{\text {DENIS }}$ as a function of $K_{\text {DENIS }}$. Shifts are 0.003 and -0.004 respectively.

\section{Obtaining star counts combining 2MASS and DENIS data}

López-Corredoira et al. 2002 (hereafter L02) obtained a model of the outer parts of the stellar galactic disc based on the 2MASS point sources survey. This model suggests a density which falls exponentially with the galactocentric radius, $R$, and with the height $z$ (variable scale-length $h_{z}(R)$ ). The scale height is variable due to the flare, which distributes the stars in a higher scale height that increases with the galactocentric distance. The model also includes the effect of the warp which produces asymmetries in the star counts. In order to estimate the accuracy in the combination of 2MASS and DENIS star counts we compare the predictions of this model, whose parameters

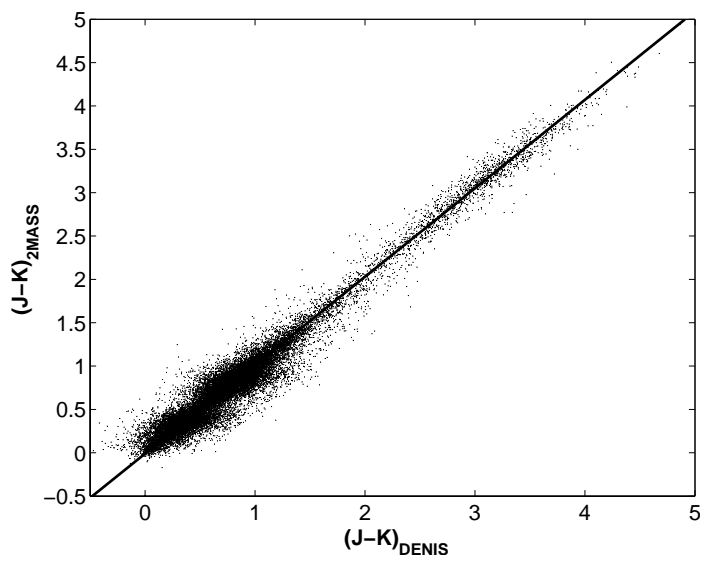

Fig. 5. $J-K_{\mathrm{s}}$ colour for $2 \mathrm{MASS}$ vs. $J-K_{\mathrm{s}}$ colour for DENIS.

have been obtained only by using 2MASS stars counts, with the combination of data from 2MASS and DENIS. Figures 7, 8. show the combination of star counts both from 2MASS and DENIS and the predictions of the L02 model. DENIS data are in good agreement with the predictions with a higher dispersion in the data due to the effect of considering less area than in 2MASS.

\section{Conclusions}

We have found that there are no significant differences between 2MASS and DENIS photometry or astrometry. No noticeable effects in obtaining star counts by combining both data sets are to be expected.

Acknowledgements. We gratefully acknowledge the referee, G. Simon, for helpful comments and suggestions that improved the final presentation of this paper. This publication makes use of data products from the Two Micron All Sky Survey, which is a joint project of the University of Massachusetts and the Infrared Processing and Analysis Center/California Institute of Technology, funded by the National Aeronautics and Space Administration and the National Science Foundation, and from DENIS, which is the result of a joint effort involving personnel and financial contributions from several Institutes, mostly located in Europe. The DENIS project has been partly funded by the SCIENCE and the Human Capital and Mobility plans of the European Commission under grants CT920791 and CT940627. It is also supported by INSU, MEN and CNRS in France, by the State of Baden-Württemberg in Germany, by DGICYT in Spain, by CNR in Italy, by FFwFBWF in Austria, by FAPESP in Brazil, by OTKA grants F-4239 and F-013990 in Hungary; and by the ESO C\&EE grant A04-046. 


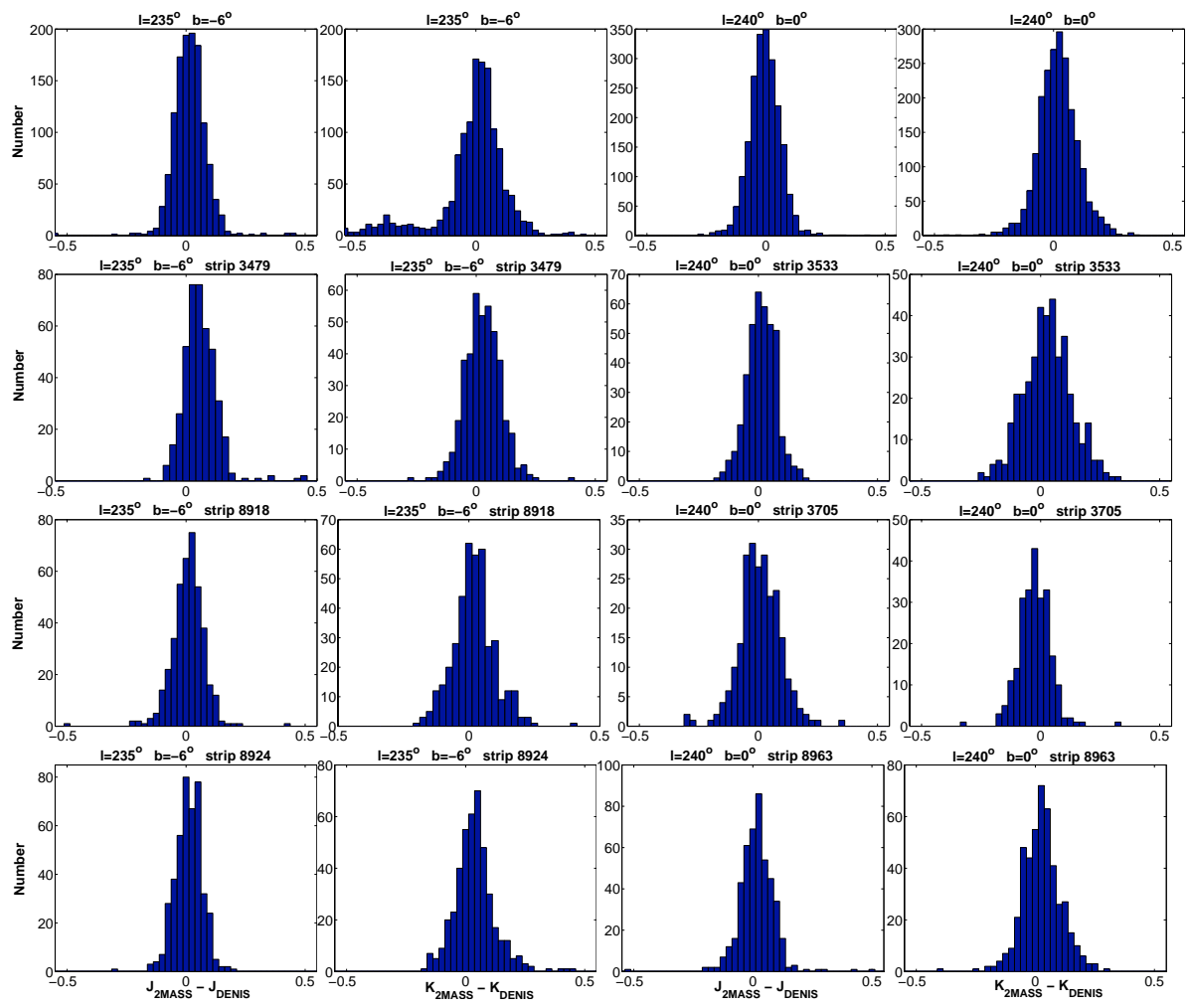

Fig. 6. Histograms of differences between DENIS and 2MASS magnitudes, for all the matched stars in the field (top panels) and including only stars from single strips (lower panels).

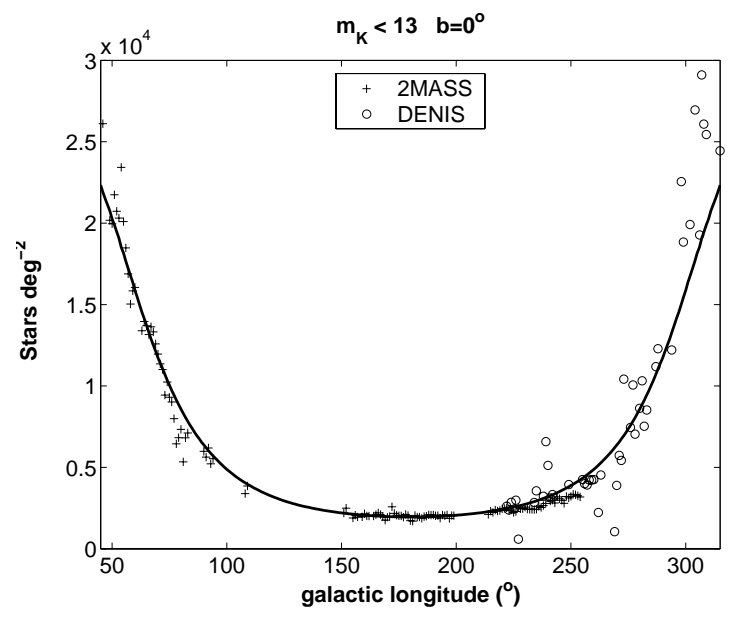

Fig. 7. Star counts in the plane by combining data from 2MASS and DENIS. Solid line is the prediction of the L02 model.

\section{References}

Carpenter, J. M. 2001, AJ, 121, 2851

Delmotte, N., Egret, D., Loup, C., \& Cioni, M. 2001, Proc. International Society for Optical Engineering, 4477, 301

Epchtein, N. 1997, in The Impact of Large Scale Near-IR Sky Surveys, ed. F. Garzón, F. N. Epchtein, N. A. Omont, B. Burton, \& P. Persi (Kluwer, Dordrecht), 15

Fouqué, P., Chevallier, L., Cohen, M., et al. 2000, A\&AS, 141, 313

Garzón, F., Hammersley, P. L., Mahoney, T., et al. 1993, MNRAS, 264,773

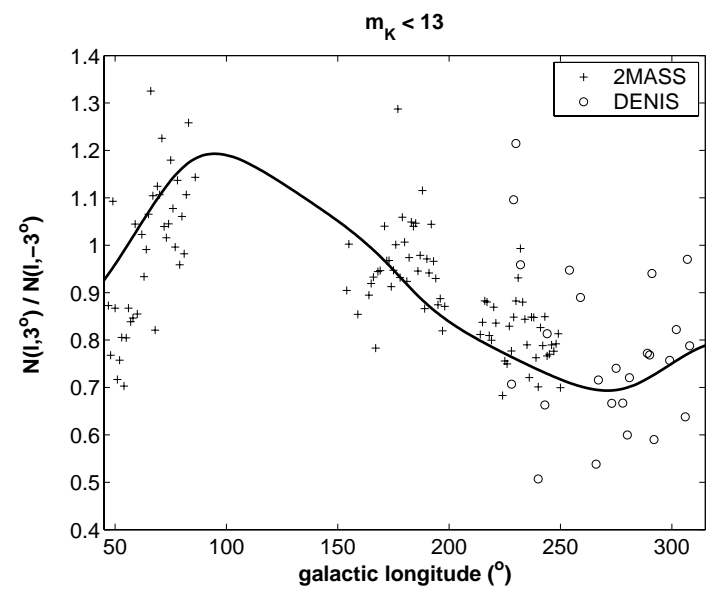

Fig. 8. Positive counts divided by the negative counts (which shows the effect of the warp) by combining data from 2MASS and DENIS. Solid line is the prediction of the L02 model.

Hammersley, P. L., Garzón, F., Mahoney, T., \& Calbet, X. 1994, MNRAS, 269, 753

López-Corredoira, M., Hammersley, P. L., Garzón, F., Simonneau, E., \& Mahoney, T. J. 2000, MNRAS, 313, 392

López-Corredoira, M., Cabrera-Lavers, A., Garzón, F., \& Hammersley, P. L. 2002, A\&A, 394, 883 (L02)

Schultheis, M., \& Glass, I. S. 2001, MNRAS, 327, 1193

Skrutskie, M. F., Schneider, S. E., Stiening, R., et al. 1997, in The Impact of Large Scale Near-IR Sky Surveys, ed. F. Garzón, F. N. Epchtein, N. A. Omont, B. Burton, \& P. Persi (Kluwer, Dordrecht), 25 